\title{
REPRESENTATIONS OF MOD $p$ LIE ALGEBRAS
}

\author{
ERIC M. FRIEDLANDER AND BRIAN J. PARSHALL
}

Let $G$ be a semisimple, simply connected algebraic group defined over an algebraically closed field $k$ of characteristic $p>0$. Because any rational $G$ module inherits the structure of a restricted module (in the sense of [4, p. 188]) for the Lie algebra $\mathcal{G}$ of $G$, the representation theory of $\mathcal{G}$ has primarily focused on the study of restricted modules. We outline here our recent investigations of the more general - that is, not necessarily restricted-represention theory of $\mathcal{G}$. Details will appear in [3].

We approach the representation theory of $\mathcal{G}$ through that of a family of finite-dimensional quotient algebras of the universal enveloping algebra $U(\mathcal{G})$ of $\mathcal{G}$. As described below, these algebras are parametrized by characters on a certain abelian subalgebra $\mathcal{O}$ of $U(\mathcal{G})$. Because the restricted enveloping algebra $V(\mathcal{G})$ appears as a distinguished member of this family (the others being thought of as "deformations" of $V(\mathcal{G})$ ), a better understanding of the representation theory of these algebras may lead to a clearer picture of that of $V(\mathcal{G})$.

For a restricted Lie algebra $\mathcal{G}$, we employ the central subalgebra $0 \subset$ $U(\mathcal{G})$ considered by Zassenhaus in his foundational paper [10]. Namely, 0 is the image of the semilinear monomorphism $S^{*}(\mathcal{G}) \rightarrow U(\mathcal{G})$ defined on the symmetric algebra $S^{*}(\mathcal{G})$ of $\mathcal{G}$ by sending $X \in \mathcal{G}$ to $X^{p}-X^{[p]}$. Using the Jordan decomposition of the dual $\mathcal{G}^{*}$ given in [7], we obtain properties such as "regular", "semisimple", or "nilpotent" for characters $\chi: 0 \rightarrow k$ whenever $\mathcal{G}=\operatorname{Lie}(G)$.

PROPOSITION 1. Let $\mathcal{G}$ be a restricted Lie algebra of dimension d and let $\chi: 0 \rightarrow k$ be a character with associated one-dimensional 0 -module $k_{\chi}$. Then $A_{\chi} \equiv U(\mathcal{G}) \otimes_{0} k_{\chi}$ is a Frobenius algebra of dimension $p^{d}$. Moreover, for each irreducible $\mathcal{G}$-module $M$, there is a unique character $\chi: 0 \rightarrow k$ such that the action of $U(\mathcal{G})$ on $M$ factors through $A_{\chi}$. g.

Of course, if $\chi=0$ then $A_{\chi} \cong V(\mathcal{G})$, the restricted enveloping algebra of

One easily proves that $\operatorname{Ext}_{U(\mathcal{G})}\left(M, M^{\prime}\right)=0$ whenever $M$ is an $A_{\chi^{-} \text {-module, }}$ $M^{\prime}$ is an $A_{\chi^{\prime}}$-module, and $\chi \neq \chi^{\prime}$. On the other hand, nontrivial computations are facilitated by the following spectral sequence.

Proposition 2. Let $\mathcal{G}$ and $A_{\chi}$ be as in Proposition 1. If $M$ and $N$ are two $A_{\chi}$-modules with $M$ finite-dimensional, then there is a natural spectral

Received by the editors November 14, 1986.

1980 Mathematics Subject Classification (1985 Revision). Primary 17B50; Secondary 17B10, 17B56.

Research supported by the National Science Foundation. 
sequence

$$
E_{2}^{s, t}(M, N)=\operatorname{Ext}_{A_{\chi}}^{s}(M, N) \otimes \Lambda^{t} \mathcal{G}^{*} \Rightarrow \operatorname{Ext}_{U(\mathcal{G})}^{s+t}(M, N) .
$$

As in our earlier work $[1,2]$ concerning the cohomology of restricted representations, we define the cohomological support variety $|\mathcal{G}|_{M}$ of a finitedimensional $A_{\chi}$-module $M$ to be the variety of the annihilator ideal in $H^{e v}(V(\mathcal{G}), k)$ of $\operatorname{id}_{M} \in \operatorname{Ext}_{A_{\chi}}^{0}(M, M)$. Most conclusions we obtain involve the image of $|\mathcal{G}|_{M}$ under the natural (finite) map $\varphi: \operatorname{Spec}\left(H^{e v}(V(\mathcal{G}), k)\right) \rightarrow \mathcal{G}$ (cf. $[1,2.1])$. For example, $M$ is a projective $A_{\chi}$-module if and only if $\varphi\left(|\mathcal{G}|_{M}\right)=\{0\} ; \operatorname{dim}|\mathcal{G}|_{M}=\operatorname{dim} \varphi\left(|\mathcal{G}|_{M}\right)$ equals the rate of growth of a minimal projective resolution of $M$ as an $A_{\chi}$-module.

Using the corresponding result for restricted representations $[2,1.4]$, we obtain the following representation-theoretic interpretation of $\varphi\left(|\mathcal{G}|_{M}\right)$.

THEOREM 3. Let $\mathcal{G}$ be a finite-dimensional restricted Lie algebra, $\chi: 0 \rightarrow$ $k$ a character, and $M$ a finite-dimensional $A_{\chi}$-module. For any $X \in \mathcal{G}$, let $\{X\}$ denote the subalgebra of $A_{\chi}$ generated by $X$. Then a nonzero element $X \in \mathcal{G}$ lies in $\varphi\left(|\mathcal{G}|_{M}\right)$ if and only if both $X^{[p]}=0$ and $M$ is not a projective $\{X\}$-module.

Clearly, $\varphi\left(|\mathcal{G}|_{M}\right)$ is a closed conical subvariety of $\mathcal{G}$. Using the above theorem, we can prove that whenever $Y \subset \mathcal{G}$ is a subvariety of $\mathcal{G}$ representable for some finite-dimensional $A_{\chi}$-module $M$ as $\varphi\left(|\mathcal{G}|_{M}\right)$, then any closed conical subvariety of $Y$ has the form $\varphi\left(|\mathcal{G}|_{N}\right)$ for a finite-dimensional $A_{\chi}$-module $N$.

We specialize to $\mathcal{G}=\operatorname{Lie}(G)$ for $G$ a simply connected semisimple algebraic group over $k$ of rank $l$ (with chosen Borel subgroup $B=T \cdot U$ containing a maximal torus $T$ and having unipotent radical $U)$. Since any $\chi: 0 \rightarrow k$ is $G$-conjugate to a character vanishing on $\mathcal{U}=\operatorname{Lie}(U)$, we restrict attention to such characters $\chi$. For each of the $p^{l}$ weights $\lambda: \not{H} \equiv \operatorname{Lie}(T) \rightarrow k$ satisfying

$$
\lambda\left(H^{[p]}\right)-\lambda(H)^{p}=\chi\left(H^{[p]}-H^{p}\right), \quad H \in \mathcal{H},
$$

we consider the "Verma-type" $A_{\chi}$-module $V_{\chi, \lambda}$ of dimension $p^{\operatorname{dim}(U)}$ induced from the one-dimensional $B=\operatorname{Lie}(B)$-module defined by $\lambda$.

For example, for $\mathcal{G}=\mathrm{sl}_{2}(p \neq 2)$, there are three $\mathrm{SL}_{2}$-orbit types of characters corresponding to $\chi$ regular semisimple, regular nilpotent, or 0 . If $\chi$ is regular semisimple, then $A_{\chi}$ is a semisimple algebra. If $\chi$ is regular nilpotent, each $V_{\chi, \lambda}$ is irreducible but only one is projective, whereas the projective covers of the nonprojective $V_{\chi, \lambda}$ are self-extensions of $V_{\chi, \lambda}$ with two composition factors. If $\chi=0$, then the $V_{\chi, \lambda}$ are indecomposable but only one is irreducible (and projective).

With the generous assistance of $\mathrm{O}$. Gabber, the authors have formulated and proved the following theorem extending a basic theorem of Kac and Weisfeiler for irreducible $A_{\chi}$-modules [6, Theorem 2]. If $A$ is a $k$-algebra, let $A$-mod denote the category of left $A$-modules.

THEOREM 4. Let $G$ be a semisimple simply connected algebraic group with Lie algebra 9 . Assume that $p$ is good for the root system of $G$ (cf. $[\mathbf{9}, p .178])$. Let $\chi: 0 \rightarrow k$ be a character with Jordan decomposition $\chi=\chi_{s}+\chi_{n}$ and let 
$\mathfrak{z}$ be the centralizer in $\mathcal{G}$ of the semisimple character $\chi_{s}$. Then $\mathfrak{z}$ is the Levi factor of a parabolic subalgebra $P=\mathfrak{z} \oplus \mathcal{N}$. Consider the functors

$$
\begin{aligned}
& \psi=(-)^{N}: A_{\chi}-\bmod \rightarrow Z-\bmod , \\
& \theta=A_{\chi} \otimes_{P}(-): Z-\bmod \rightarrow A_{\chi}-\bmod
\end{aligned}
$$

where $Z$ (respectively, $N, P$ ) denotes the subalgebra of $A_{\chi}$ generated by $\mathfrak{z}$ (resp., $\mathcal{N}, P)$. Then $\theta$ is left adjoint to $\psi$, both $\theta$ and $\psi$ are exact functors, and the adjunction morphisms $\mathrm{id}_{Z \text {-mod }} \rightarrow \psi \circ \theta$ and $\theta \circ \psi \rightarrow \mathrm{id}_{A_{x}-\bmod }$ are isomorphisms.

The preceding theorem relates the study of the representation theory of $A_{\chi}$ for an arbitrary character $\chi: 0 \rightarrow k$ to that of the representation theory of a possibly smaller Lie algebra $\left(\mathfrak{z}^{\prime}=[\mathfrak{z}, \mathfrak{z}]\right.$ in the above theorem) and a nilpotent character (the restriction of $\chi_{n}$ to $\mathfrak{z}^{\prime}$ ). For example, we readily obtain a strengthening of a result of Rudakov [8] concerning minimal projective $A_{\chi}$-modules, as well as necessary and sufficient conditions for $A_{\chi}$ to be a semisimple algebra. In conjunction with Proposition 2, this yields an explicit calculation of $\operatorname{Ext}_{U(\mathcal{G})}(M, N)$ for "most" irreducible $U(\mathcal{G})$-modules $M, N$.

In seeking to generalize the example of $\mathrm{sl}_{2}$ discussed above for regular nilpotent characters $\chi: 0 \rightarrow k$, the authors have investigated the suggestive discussion of Kac in [5]. We obtain the following theorem.

THEOREM 5. Let $G$ be a simply connected simple algebraic group of type $A_{l}, B_{l}, C_{l}$ or $G_{2}$ and let $\mathcal{G}=\operatorname{Lie}(G)$. Assume that $p$ does not divide the order of the quotient of the weight lattice by the root lattice. For any regular nilpotent character $\chi: 0 \rightarrow k, V_{\chi, \lambda}$ is an irreducible $A_{\chi}$-module.

Using Theorem 5, we can obtain much useful information concerning the representation theory of $A_{\chi}$ for $\chi$ regular nilpotent. We employ the notation $\sim$ for the equivalence relation on the set $X$ of integral weights $\lambda: \forall \rightarrow k$ defined by $\lambda \sim \mu$ if and only if $w(\lambda+\rho)=\mu+\rho$ for some element $w$ of the Weyl group $W$ of $G$ (where $\rho$ is the half-sum of the positive roots).

THEOREM 6. Let $G$ and $p$ be as in Theorem 5 and assume that $\chi$ is a regular nilpotent character. Let $S_{\lambda}$ denote the irreducible $A_{\chi}$-module $V_{\chi, \lambda}, \lambda \in$ $X$.

(a) For $\lambda, \mu \in X$, $\operatorname{Ext}_{A_{\chi}}\left(S_{\lambda}, S_{\mu}\right) \neq 0 \Leftrightarrow \lambda \sim \mu \Leftrightarrow S_{\lambda} \cong S_{\mu}$.

(b) The projective cover of $S_{\lambda}$ as an $A_{\chi}$-module has a composition series with $|\{\mu \in X: \lambda \sim \mu\}|$ factors, each isomorphic to $S_{\lambda}$.

(c) $S_{-\rho}$ is the unique (up to isomorphism) projective, irreducible $A_{\chi}$-module.

\section{REFERENCES}

1. E. Friedlander and B. Parshall, Geometry of p-unipotent Lie algebras, J. Algebra (to appear).

2. __ Support varieties for restricted Lie algebras, Invent. Math. 86 (1986), 553-562.

3. __ Modular representation theory of Lie algebras (to appear).

4. N. Jacobson, Lie algebras, Interscience, 1962.

5. V. Kac, Irreducible representations of Lie algebras of classical type, Uspekhi Mat. Nauk 27 (1972), no. 5, 237-238. (Russian) 
6. V. Kac and B. Weisfeiler, Irreducible representations of Lie p-algebras, Funktsional. Anal. i Prilozhen, 5 (1971), no. 2, 28-36. (Russian)

7. _ Coadjoint action of a semisimple algebraic group and the center of the enveloping algebra in characteristic $p$, Indag. Math. 38 (1976), 135-151.

8. A. Rudakov, On the representation of the classical Lie algebras in characteristic $p$, Izv. Akad. Nauk SSSR 34 (1970), 735-743. (Russian)

9. T. Springer and R. Steinberg, Conjugacy classes, Lecture Notes in Math., vol. 131, Springer-Verlag, Berlin and New York, 1970, pp. 167-266.

10. H. Zassenhaus, The representations of Lie algebras of prime characteristic, Proc. Glasgow Math. Assoc. 21 (1954), 1-36.

Department of Mathematics, North Western University, Evanston, ILLINOIS 60201

Department of Mathematics, University of Virginia, CharlottesVILLE, VIRGINIA 22903 